\title{
STEM CELL THERAPY FOR DEMYELINATING DISORDERS
}

\author{
Jan Pazour, Jaroslav Mokrý
}

Charles University in Prague, Faculty of Medicine and University Hospital in Hradec Králové, Czech Republic: Department of Histology and Embryology

Summary: Multiple sclerosis (MS) is a progressive disease of the central nervous system (CNS) that attacks mainly young people. It leads to the progressive deterioration of the neurological status. Histopatologically, this disease is characterized by appearance of multiple foci of the demyelination in white matter of the CNS, with various grade of an axonal loss. The current treatment is targeted on moderating the inflammatory process and symptomatic therapy. In spite of all this therapy, the course of the disease often progresses. The tissue of the CNS in mammalians, including humans, is able to provide some degree of spontaneous remyelination. Unfortunatelly the extent of this process is not sufficient for the complete restoration. The support of remyelination by using the cell manipulations is the aim of many experimental studies. Theoretically, it is possible to achieve remyelination either by exogenous induction of remyelination from endogenous sources (precursor cells) or by the real transplantation of myelin-forming cells intrafocally, intracerebroventricularly or into the blood stream. In this work, we present the brief view on the recent state of this topic. We present the list of the cell types, useable for cell transplantations and the summary of the growth factors influencing the behaviour of the oligodendroglial precursors. We are considering the hampers in usage of the cell therapy of demyelinating disorders in clinics.

Key words: Demyelinating disease, Remyelination, Cell therapy, Oligodendroglial progenitor cells, EAE, Growth factors

\section{Introduction}

Multiple sclerosis is the main member of the group of demyelinating diseases. This human disorder is characterized by the loss of myelin, myelin-forming cells and axons of the central nervous system. In many cases, it leads to the progressive neurological deterioration. The disease usually begins between 20-30 years of age. Women suffer two-times more frequently than men. The prevalence in our geografical region is about $50-100$ per 100,000 inhabitants. The etiology seems to be multifactorial, comprising the interaction between the exogenous and endogenous factors, which trigger the cascade of biological actions. The initiation of the inflammatory process in the CNS is connected with damage of the nervous tissue caused either by infection or by impaired apoptosis. Macrophages and microglia identify endogenous and exogenous dangerous molecules through their membrane receptors. They analyze the autoantigens and present them to T-cells. They also produce pro-inflammatory cytokines (TNF alpha, IL-1 beta) and chemokines (MCP-1, MIP-1 alpha, MIP 1 beta, IP10, Rantes) that affect blood brain barrier characteristics. The adhesivity of endothelial cells is upgraded for immune system cells that transmigrate in higher amount to the CNS compartment. Migration of immune cells is directed by the chemokines.

Subset TH 0 lymphocytes transforms to the subset TH 1, following by INF gamma production. INF gamma increases the expression of proapoptotic molecules on the cells of CNS. T cells are also the source of glutamate that binds on its receptors on the surface of oligodendrocytes and neurons and so causes the damage of these cells.

Macrophages are important effector cells directly harming the myelin sheaths. Apoptosis is induced in the cells of the CNS through TNF alpha. This process is stimulated by binding of TNF alpha on its receptors p55 and p75. Apoptosis can be stimulated also by intracellular molecules, so called granzymes produced by cytotoxic cells. It leads to activation of the intracellular proteolytic enzymes - caspases, followed by the activation of the nucleases that deteriorate the cells.

All these mechanisms result in demyelination, axonal and glial damage. They contribute to the development of various neurological signs, dependent on the extent and localization of the pathological changes in the CNS.

Histopathologically, the disease is characterized by formation of multiple foci of demyelination in the white matter of the CNS with various grade of an axonal loss. In the acute lesion, the plaque, we can find perivascular infiltrates of lymfocytes and macrophages, massive loss of myelin sheaths and various degree of axonal transsection. In the chronic plaques, demyelination and the axonal loss predominate. On the contrary, the inflammatory component decreases.

Current treatment is based on the application of the anti-inflammatory drugs, physiotherapy and symptomatic 
therapy. Recently, the high doses of interferon beta are administered to prevent new attacks and progression of the disease. Unfortunatelly, there is still often significant progression in spite of this complex therapy.

In recent years, cell therapy constitutes a new promise in the treatment of demyelinating diseases. This approach is based either on an activation of remyelination from the endogenous pool of myelin-forming cells or on the transplantation of exogenous cells, that are able to migrate, differentiate into myelin-forming cells and myelinate the injured axons. The successful remyelination would lead to the clinical improvement. Many promising experimental outcomes in this area were achieved, but many questions are not still answered.

\section{Animal models of demyelination}

1. The demyelination induced by toxins includes models induced by injection of the gliotoxic chemicals like lysolecithin or ethidium bromide, into the white matter of the CNS. It leads to the loss of the myelin localized to the area around the stitch. Oral administration of cuprizone is also described (39). The course of the experimental disorder doesn't imitate the human disease.

2. Experimental autoimmune encephalomyelitis (EAE) EAE represents the most often used model of demyelination in the experiments. EAE is achieved either by the active immunisation with the CNS homogenate, myelin proteins like myelin oligodendrocyte glycoprotein (MOG), myelin basic protein (MBP) in complete Freund adjuvans injected subcutaneously or by passive transfer of activated $\mathrm{CD}^{+}$T-cells isolated from spleens or lymph nodes of animals that are actively immunized with encephalitogenic antigen (MBP). It leads to multifocal involvement of the white matter by inflammation, demyelination and destruction of axons. Models of EAE best imitate the course of MS in humans.

Acute EAE (aEAE) is the monophasic disease, which develops usually 10-12 days after immunization and recovers spontaneously 18-20 days after immunization. Histopatologically focuses of inflammation predominate. There is lower degree of demyelination. In opposite chronic EAE is characterized by extensive demyelination of the CNS with axonal damage and irreversible neurological deficit.

3. Viral demyelination models

The most common is the Theiler's murine encephalomyelitis (TMEV). The Theiler's virus is the neurotrophic picornavirus. CD $4^{+}$lymphocytes react specifically with the viruse's antigens. Later the immunopathological process extends to other chemical structures like proteolipid protein (PLP). The clinical course and histology is similar to the chronic progressive MS in humans.

4. Other models

Myelin deficient (md) rats carry the gene defect for PLP. Shaking (sh) pups, the canine model of the PLP defici- ency. Shiverer (shi) mice carry the gene defect for MBP. These gene mutations lead to the faulty myelin formation.

\section{Development of oligodendroglial cell lineage}

The myelin-forming cells of the CNS rise from the oligodendroglial progenitors (OPCs). Knowlege of development of this cell line is therefore very important for the study of the cell therapy of demyelinating disorders.

The source of oligodendroglia are multipotential neural stem cells (NSCs), occuring in various areas of the fetal and adult CNS, including ventricular and subventricular zone, external germinal zone of cerebellum, subgranular zone of dentate gyrus (17). Progenitor cells constitute the next step in the differentiation of NSCs. They are committed to the development to specific cell line. It remains unclear, how irreversible this differentiation is. Under the defined conditions in vitro, oligodendroglial progenitors are able to produce astrocytes and oligodendrocytes (5).

Oligodendrocytes come through several developmental stages characterized by expression of specific markers (gangliosid GD3, the surface antigen A2B5, O4 antigen, AN2 glycoprotein, CNPase, transcription factors Olig 1, Olig 2, integral membrane proteoglycan NG2 etc.). Detection of these antigens allows recognizing the developmental stages in experiments.

Bipotent cells isolated from the neonatal optic nerve were called $\mathrm{O}-2 \mathrm{~A}$ progenitors, which migrate there from chiasma opticum (28). The term O-2A results from the fact, that they are able to differentiate in vitro either into oligodendrocytes or astrocytes type II. Direction of the development depends on the enviromental conditions, for example on the concentration of platelet derived growth factor-alpha (PDGF-alpha) in growth medium. In the PDGF free medium, they differentiate into oligodendrocytes, while in the presence of this factor they give rise into astrocytes type II. PDGF alpha is produced by another cell line, astrocytes type I, which are therefore the potent mitogens for O-2A progenitors. The next factor supporting the $\mathrm{O}-2 \mathrm{~A}$ maturation into astrocytes is ciliary neurotrophic factor (CNTF).

The initial stages of OPCs development exhibit $\mathrm{NG}^{+}$, PDGF- $\mathrm{R}^{+}, \mathrm{O} 4-$ phenotype. These cells give rise to more advanced progenitors, which exhibit $\mathrm{O} 4$ antigen. The next step in OPCs maturation are premyelinating oligodendrocytes, which lose NG-2 and acquire proteolipid protein (PLP) expression. The mature myelinating oligodendrocytes express MBP, CNPase, MOG and Gal-C. The O4 expression is the first step to the terminal differentiation of oligodendrocytes (Table 1).

The adult OPCs differ from their prenatal counterparts in the rate of cell division, migration and differentiation, which are 3-4 times lower in adults (20). There are several mechanisms that lead to this deceleration. One possible mechanism is the cumulation of the $\mathrm{p} 27 \mathrm{Kipl}$ cell cycle inhibitor. The activation of the Notch receptor on OPCs is 
the next possible blocker of the OPCs differentiation. Bone morphogenic protein (BMP) inhibits the differentiation of OPCs into oligodendrocytes through its receptor on OPCs, while it supports the maturation into astrocytes (18). Insulin like growth factor 1 (IGF-1) and neurotrophin 3 (NT3) induce the proliferation of OPCs (4).

The OPCs migratory capacity is affected by various factors like PDGF, chemokine CXCL1, semaphorins (sema A, sema $F$ and netrin-1). It is also established that the duration of the OPCs cell cycle differs in distinct areas of the CNS. For example cortical OPCs are more quiescent then OPCs derived from the optical nerve (34).

\section{Endogenous remyelination}

Today it is clear, that there are areas in the adult CNS containing neural stem cells. NSCs participate in the development of the CNS by proliferation and differentiation in various cell types. Postnatally their mitotic activity decreases and most NSCs become quiescent and form the pool for all cell lineages of the CNS that can replace the lost neural cells.

NCSs were found mainly in the rostral part of the subependymal zone of the CNS. Subependymal zone (SEZ) is the complex of several cell layers adjacent to the ependyma of the lateral ventricles. This zone comprises several cell types:

1. Neuroblasts migrating through the rostral migratory stream (RMS) to olfactory bulb, where they replace the abolished neurons of the granular and glomerular layer.

2. Nestin ${ }^{+}, \mathrm{GFAP}^{+}$astrocytes

3. Nestin ${ }^{+}$stem cells

4. Ependymal cells lining lateral ventricles and central canal of the spinal cord. According to Johansson et al. these cells can display stem cell like qualities (21). It is considered, that these elements could be the cell residuals of the embryonic ventricular zone. They are also responsible for the glial scare formation after trauma of the nervous tissue (6).

During the course of EAE increased cell proliferation occurs in the SEZ. It indicates, that the progenitors in this area proliferate in response to inflammation or demyelination (12). It is not clear, if the proliferation in the SEZ is promoted rather by inflammation or by demyelination. Studies comparing models of demyelination combined with inflammation and on the contrary the inflammation without demyelination show stronger response of the SEZ in the demyelinating models (15). On the other hand, growth and mitogenic factors released during inflammation can increase the number of OPCs. These data underline importance of the inflammatory microenvironment on the activation of endogenous progenitors (6).

Recently it has been evidenced, some degree of spontaneous remyelination really occurs in the lesioned CNS tissue. However, the potential for repair is very limited for several reasons. In acute lesions, higher density of oligo- dendrocytes was identified, while in chronic lesions, there was a lack of myelin-forming cells and no signs of remyelination (9). These facts indicate, that on the beginning of the disease endogenous remyelination could be effective, while this ability is exhausted in the chronic phase and it leads to the permanent loss of the myelin-forming cells and the failure of remyelination.

It was also established, that the mature oligodendrocytes survived in the lesions but they were not able to remyelinate demyelinated areas of the CNS (23). Therefore remyelination is performed by the local oligodendrocyte progenitor cells, migrating to the lesions and differentiating into the myelin-forming cells. However remyelinating capacity of these cells is low. Limiting factor is the reduced ability of cells to migrate into the lesioned sites. Only cells localized in the immediate neighbourhood migrate in the lesioned tissue. Regarding to the disability of OPCs self-renewal, the local pool of progenitor cells is exhausted very soon, which leads to the definitive failure of remyelination. These findings support the hypothesis, that the effectiveness of remyelination is higher in acute lesions with more intensive inflammatory response (33). Key role in this process play cytokines IL-1 beta and TNF alpha (27). The anti-inflammatory treatment could be the reason, why endogenous remyelination fails. Another cause of the endogenous remyelination failure is the astroglial scarring. It restrains the entrance of the myelin-forming cells to the lesion. Astrocytes also produce number of growth factors influencing OPCs proliferation and differentiation (34). Neuregulins produced by astrocytes are decreased in active MS lesions. It could be the reason of impaired development of OPCs (40). In addition astrocytes in demyelinated lesions activate Notch-1 receptor on OPCs through the Jagged-1 ligand expression and thereby they inhibit maturation of OPCs. The Notch-1 activation could be mediated by cytokine TGF beta 1 , which is upregulated in MS (22).

The next factor influencing long-term survival of the progenitors is the bi-directional trophic interaction between the precursors and axons. Multiple axonal transsections that appear in MS could restrain successful remyelination (21). Therefore it is necessary to provide the myelin restoration as soon as possible, before the massive axonal damage appears. This time window is very close.

It is suppossed, that the combination of environmental factors and the basic characteristics of oligodendroglial cells prevent the successful endogenous remyelination. More detailed understanding of the influence of these issues has to be the matter of the future research.

\section{Induction of remyelination}

There are two main approaches, how to achieve remyelination in demyelinating disorders:

\section{Factors facilitating myelination}

The aim of this approach is to increase the capacity of self-repair of the CNS. Recently, there were identified seve- 
ral growth factors, that are able to support proliferation, viability and differentiation of the OPCs. The important factors in this process are neurotrophins, IGF-1, FGF, PDGF, cytokines of the neuropoetin's family and neuregulins.

\section{Neurotrophins}

Neurotrophins constitute the important factors for the development and function of the nervous system. This group includes nerve growth factor (NGF), brain derived neurotrophic factor (BDNF), neurotrophin 3 and 4 (NT 3,4). Among others they affect the oligodendroglial lineage development. NT-3 supports in vitro survival and proliferation of OPCs, while BDNF and NGF protect OPCs against cell death caused by pro-inflammatory cytokines like TNFalpha (3). Neurotrophins also downregulate pro-inflammatory activity of macrophages and microglia. It was established in experiments, that NGF prevents EAE development through its anti-inflammatory effects (3).

\section{Insulin-like growth factor-1 (IGF-1)}

IGF-1 is involved in regulation of proliferation and differentiation of OPCs. Among others it protects immature glial progenitors against the cell death induced by glutamate and other demyelinating chemicals. Application of IGF-1 to animals suffering from EAE reduces the extent of demyelinated lesions and supports remyelination process (41). However administration of recombinant IGF-1 to several MS human patients has not led neither to clinical nor magnetic resonance imaging improvement (16).

\section{Platelet derived growth factor (PDGF)}

PDGF has promigratory and mitogenic effects on OPCs. It supports survival of the progenitors as well. There is a correlation between intralesional level of PDGF and the amount of OPCs. Moreover, PDGF also supports recruitment of these cells into the lesion.

\section{Fibroblast growth factor (FGF)}

FGF family is the greatest group of growth factors influencing the mesodermal and ectodermal cells. Basic FGF (b-FGF) exhibits promigratory and mitogenic effects on OPCs. It also blocks differentiation of OPCs into mature oligodendrocytes by increasing number of PDGF receptors on OPCs. Intracerebral administration of FGF in experiment has shown the protective effect on EAE progression and supports the endogenous remyelination through the increased number of OPCs within the lesions (36).

\section{Neuropoetins}

Neuropoetins are the group of cytokines, which is engaged in the inflammatory processes. These factors are activated during the damage of the CNS tissue as well as during the reparative phase. Leukemia inhibitory factor (LIF) and ciliary neurotrophic factor (CNTF) support survival, proliferation and differentiation of OPCs. They also protect OPCs against cell death caused by pro-inflammatory cyto- kines like interferon-gamma and TNFs. In EAE model, exogenously administered LIF ameliorated signs of the disease and also played a role in limiting an oligodendrocyte loss during autoimmune attack through LIF receptor-beta signalling (11). Studies on CNTF-deficient mice also support a role for CNTF in protecting the oligodendrocytes against the cell death induced by inflammation (25).

\section{Neuregulins}

Neuregulins is a group of ligands exhibiting the trophic effects on neurons and glia. Glial growth factor-2 (GGF-2) supports survival and proliferation of OPCs and prevents their differentiation. In experimental model, administration of recombinant human GGF-2 to mice with EAE ameliorated clinical as well as histopatological signs of the disease (13).

The following research of the effects of growth factors should give new fragments of information, how to initiate neural precursors to produce OPCs that are able to migrate and produce myelin-forming cells.

\section{Cell transplantation}

The transplantation of myelin-forming cells is a mode of delivering the entire „cell factory“ that manufactures myelin (6). It is the essential procedure for cell therapy of demyelinating diseases. The aim is to deliver cells, which are able to myelinate demyelinated axons within the lesions and thus improve conductivity of axons followed by clinical improvement.

Recently, promising outcomes in this field were achieved by many researchers. However, many problems remain unsolved. What kind of cells is the best for transplantation? In which phase of their differentiation they should be transplanted? Other relevant factors that could influence the success of the therapeutic procedure are timing of transplantation, route of cell delivery etc. It is clear, that multifocal involvement, as we find in MS, prevents the possibility of intrafocal cell transplantation.

The cell types that were tested in transplantation experiments are listed below.

\section{Cells of oligodendroglial lineage}

The experimental transplantation of oligodendrocytes has shown that the ability of differentiated oligodendrocytes for remyelination is very poor (14). On the other hand, OPCs have major remyelinating potential. Exogenous OPCs had even higher myelinating ability than endogenous cells (8). Interesting results were described after intrafocal transplantation of purified $\mathrm{O}-2 \mathrm{~A}$ progenitors, which were expanded in vitro with growth factors PDGF and FGF beta. Three weeks post transplantation, 8 of 10 experimental animals exhibited unequivocal signs of repair. In 4 of 8 animals, remyelination was verified microscopically in more than $90 \%$ of axons (19). Another study accents the infuence of inflammatory enviroment on the survival of transplanted oligodendroglial progenitors. In experimental rats, the high 
number of viable transplanted cells was detected even after 50 days post transplantation. The cells were found to $6 \mathrm{~cm}$ far from the area of instilation. In opposite, in control group without demyelination, migratory properties of transplanted cells was minimal. This can be explained by the absence of growth and chemotactic factors (PDGF, IGF-1, CNTF, NT-3) in unaffected tissue (38). It supports the idea, that anti-inflammatory treatment for MS can complicate the success of the cell therapy.

Tab. 1: The developmental stages of OPC's differentiation and expression of the antigens.

\begin{tabular}{|l|l|}
\hline OL progenitor & $\begin{array}{l}\text { NG-2 +, PDGF-R + } \\
\text { A2B5, Vimentin }\end{array}$ \\
\hline Pre OL & $\begin{array}{l}\text { O4, NG2, A2B5, } \\
\text { Vimentin, PDGF-R }\end{array}$ \\
\hline Immature OL & CNP ase, MAG, \\
& GalC, O4 \\
\hline Mature OL & $\begin{array}{l}\text { MBP, CNP-ase, } \\
\text { MOG, Gal-C, } \\
\text { PLP, MAG }\end{array}$ \\
\hline
\end{tabular}

Tab. 2: Antigens expressed by various cell types.

\begin{tabular}{|l|l|}
\hline Oligodendrocyte & MBP, PLP, MOG, CNP ase, GalC \\
\hline OEC & GFAP, O4, p75 NGF receptor \\
\hline Schwann cells & P75 NGF receptor \\
\hline
\end{tabular}

\section{Schwann cells}

Schwann cells are the myelinating elements of the peripheral nervous system. They differ from oligodendrocytes in their morphology and in the way, how they produce the myelin. A single Schwann cell myelinates only one peripheral axon in contrast to the oligodendrocyte, that myelinates more axons. Schwann cells express on their surface different antigens than oligodendrocytes (Table 2).

Although Schwann cells are peripheral nervous elements, it has been established, that they exhibit good myelinating capacity in the CNS (7). In the experimental work, the transplantation of Schwann cells in demyelinated rats resulted in remyelination of dorsal funiculus and improvement of the conduction velocity in the restored nerve fibres (24). Interestingly, the Schwann cells transplantation did not lead to the invasion to the surrounding nervous tissue out of the lesion site. In transplantation experiments with this type of cells, neither the invasive growth nor tumourigenic behaviour was detected.

It is also advantageous, that it is possible to use these cells as the autologous transplants. They may be obtained from the peripheral nerves (sural nerve) and cultivated in vitro. Hypothetically these cells, as the peripheral elements, could obviate the autoimmune attack targeted against the myelin-forming cells of the CNS.

The clinical study with the Schwann cell transplantation in MS patients was performed in Yale University in 2001-2002. The autologous transplantation of Schwann cells was performed into single demyelinating lesion. Unfortunatelly the study was not successful and was interrupted. Five month after transplantation there were no signs neither of survival of the cells nor of remyelination in the specimens obtained from biopsies.

Olfactory nerve ensheathing cells (OECs)

Olfactory nerve ensheathing cells are pluripotent cells belonging to the peripheral olfactory system and are closely located to axons of the first cranial nerve. Immunohistochemically they display characteristics of astrocytes (GFAP expression), Schwann cells (p75) and oligodendrocytes (O4) (31). Although normally these cells do not produce myelin but after transplantation they are able to myelinate axons with the myelin morphologically similar to the myelin produced by Schwann cells (42). Xenotransplanted canine or human OECs isolated from the adult olfactory bulb have been capable of extensive functional remyelination after the transplantation into demyelinated CNS of rats (36). These cells also support the growth of axons and produce neurotrophic factors (26). It is not yet clear, if these cells can be obtained from extracranial sites or only from the olfactory bulb.

\section{Neural stem cells (NSCS)}

Neural stem cells are multipotent elements found out in the fetal as well as adult CNS. During the development they serve as a source of cells for the building-up of the CNS. In adulthood, they form the pool of stem cells for compensation of the cell's lost and for the tissue repair. NSCs are able to differentiate into three basic cell lineages: neurons, oligodendrocytes and astrocytes.

NSCs can be isolated from both fetal and adult mammalian brain and cultured in chemically defined medium substituted with EGF and bFGF. Under these conditions, NSCs proliferate and form three-dimensional multicellular neurospheres. By the dissociation, it is possible to passage neurosphere cells repeatedly and obtain the great amount of undifferentiated neural stem cells. The transfer of the cells on the adhesive substrate and removal of growth factors leads to their differentiation into neuronal, astroglial and oligodendroglial cells (28).

NSCs are considered as a promising substrate for the cell therapy of demyelinating disorders. They have higher migratory capacity to the lesioned sites, than more differentiated OPCs. It is also easier to obtain a great number of elements by culturing in vitro. It is likely, that microenviromental factors influence the differentiation of NSCs to desirable direction. The transplantation of NSC intrathecally or intravenously in experimental models of EAE led to successful migration of cells into the multiple lesions and to their differentiation into myelin-forming cells. The reduction of reactive astrogliosis and axonal loss was also observed (30). It has been supposed, that transplanted cells may circulate in the blood stream or cerebrospinal fluid before they identify the inflammation focus where they cross 
the blood-brain barier. The way, how they detect the impaired tissue is probably mediated by the number of signal molecules that are recognized by transplanted cells. It seems that the inflammatory component of the demyelinating disease is very important for cell migration and integration in the tissue.

Unconvincing results were decribed after intracerebroventricular transplantation of NSCs to MAG/fyn deficient mice. The majority of donor cells formed the clusters that remained in the cerebral ventricles. The analysis of the clusters showed that they are formed mainly by astrocytes. Only few cells migrated into lesion sites and produced myelin. In opposite, cell transplantation directly into the hypomyelinated white matter of the spinal cord led to widespread differentiation of cells followed by remyelination (1). These results underline the importance of enviromental factors on the behaviour of transplanted cells.

The clinical improvement was noted after the transplantation of NSCs in the animal model of the stroke and spinal trauma as well (37).

It remains unclear, which degree of the cell differentiation is optimal for successful transplantations. Also the best route of administration of donor cells is not defined.

\section{Embryonic Stem Cells (ES)}

ES cells derived from the inner cell mass of the blastocyts are considered as pluripotent cells capable of generating all cell types of the organism. These cells can be directed to differentiation in desired phenotype in vitro. For example, the glial precursors were generated from mouse ES under the subsequent treatment with growth factors bFGF, EGF and PDGF (10). Recent studies show the possibility to achieve high purified oligodendroglial cultures from ES cells, capable to integrate and differentiate into myelinforming oligodendrocytes after the transplantation into the spinal cord of experimental mice (29). The main danger threatening after the ES cell transplantation is formation of teratomas (43). The cell therapy of demyelinating disorders using ES cell derivatives may be possible only after a careful and reliable removal of all undifferentiated ES cells.

Bone Marrow Stromal Stem Cells (BMSSCS)

BMSSCs represent the second type of stem cells of the bone marrow next to the haemopoietic stem cells. They show the high degree of plasticity, i.e. ability of transdifferentiation in various cell types. Thanks to these characteristics BMSSCs are now intensively studied. These cells can be also expanded in vitro. The transplantation of BMSSCs either into the spinal cord or by the intravenous infusion led to remyelination in the rat model of demyelination (2).

\section{Conclusion}

Till this time, it is not clear, which strategy of the cell therapy for demyelinating disorders is optimal. More alternatives have been suggested. The support of remyelination from the endogenous sources is possible with various growth factors and cytokines (GGF-2, IGF-1, LIF, EGF, FGF etc.). The success of this approach is dependent on whether the endogenous precursor cells were affected with the disease. Another possibility is the transplantation of various cell types resulting in their differentiation in the myelin-forming cells. If the disease affects the microenviroment, which becomes non-permisive for myelin-forming cells, then this approach is problematic as well. Under such conditions, the transplanted cells have to be resistant against the enviroment.

The next unresolved question is what route of cell administration is optimal for cell transplantations. It was mentioned above, that in many experiments the promising results were achieved after the intrafocal transplantation of various cell types, which led to long-standing cell survival and extensive remyelination of the lesions followed by the clinical improvement. However, this approach is unfortunatelly hardly usable in MS, characterized by the multifocal impairment of the CNS. The promising results were described after intracerebroventricular and intravenous transplantation of NSCs (30). This approach seems to be more realistic for the cell therapy of MS. However, there are still unsolved problems like insuficient migration, integration and long-lasting survival of transplanted precursor or stem cells. The important task is also the assessment of the optimal timing of the procedure. It's clear that the transplantation must be performed as soon as possible in the acute phase of the disease before the extensive axonal damage, which hampers successful remyelinating process.

Cell transplantation has to be safe and not tumourigenic. The solution of all these questions can be found in continuous research of the pathogenesis of the demyelinating disorders, as well as the studies of factors, influencing the precursor cells biology and behaviour.

\section{Acknowlegment}

This work is supported by the grant from IGA Ministry of Health Czech Republic, NR/7969-3

\section{References}

1. Ader M, Schachner M, Bartsch U. Integration and differentiation of neural stem cells after transplantation into the dysmyelinated central nervous system of adult mice. Eur J Neurosci 2004;20:1205-10.

2. Akiyama Y, Radtke C, Honmou O, Kocsis JD. et al. Remyelination of the spinal cord following intravenous delivery of bone marrow cells. Glia 2002;39:229-36. Aloisi F. Growth factors. Neurosci 2003;24:291-4

4. Barres BA, Schmid R, Sendnter M, Raff MC. Multiple extracellular signals are required for long-term oligodendrocyte survival. Development 1994;118:371-5.

5. Ben-Hur T.,Rogister B, Murray K, Rougon G, Dubois-Dalcq M. Growth and fate of PSA NCAM ${ }^{+}$precursors of the postnatal brain. J Neurosci 1998;18:5777-88.

6. Ben-Hur T, Einstein O, Mizrachi-Kol R.et al. Stem cell therapy for myelin diseases. Current Drug Targets 2005;6:3-19.

7. Blakemore WF, Crang AJ. The use of cultured autologous Schwann cells to remyelinate areas of persistent demyelination in the central nervous system. J Neurol Sci 1985;70:207-23.

8. Blakemore WF, Gilson JM, Crang AJ. Transplanted glial cells migrate over a greater distance and remyelinate demyelinated lesions more rapidly than endogenous remyelinating cells. J Neurosci Res 2000;61:288-94.

9. Bruck W, Schmied M, Suchanek G. Oligodendrocytes in the early course of multiple sclerosis. Ann Neurol 1994;35:65-73. 
10. Brustle O, Jones KN, Learish RD.et al. Embryonic stem cell-derived glial precursors: a source of myelinating transplants. Science 1999;285:754-6.

11. Butzkneven H, Zhang JG,Soilu-Hanninen M. et al. LIF receptor signaling limits immune-mediated demyelination by enhancing oligodendrocyte survival. Nat Med 2002;8:613-9

12. Calza L, Fernandez M, Giuliani A, Aloe L, Giardino L. Proliferation and phenotype regulation in the subventricular zone during experimental allergic encephalomyelitis. Proc Natl Acad Sci USA 1998;95:3209-14.

13. Cannela B, Hoban CJ, Gao et al. The neuregulin, glial growth factor-2, diminishes autoimmune demyelination and enhances remyelination in a chronic relapsing model for multiple sclerosis. Proc Natl Acad Sci USA 1998;95:10100-105.

14. Crang AJ, Gilson J, Blakemore WF. The demonstration by transplantation of very restricted remyelinating potential of post-mitotic oligodendrocytes. J neurocytol 1998;27:541-53

15. Di Bello IC, Dawson MR, Levine JM, Reynolds R. Generation of oligodendroglial progenitors in acute inflammatory demyelinating lesions of the rat brain stem is associated with demyelination rather than inflammation. J Neurocytol 1999;28:365-81.

16. Frank JA, Richert N, Lewis B. et al. A pilot study of recombinant Insuline- like growth factor-I in seven multiple sclerosis patiens. Mult Scler 2002;8:24-9.

17. Gage FH, Kempermann G, Plamer TD, Peterson DA, Ray J. Multipotent progenitor cells in the adult dentate gyrus. J Neurobiol 1998;36:249-66.

18. Gross RE, Mehler MF, Mabie PC, Zang Z, Santschi L, Kessler JA. Bone mor phogenic proteins promote astroglial lineage commitment by mammalian subventricular zone progenitor cells. Neuron 1996;17:595-606.

19. Groves AK, Barnett SC, Franklin RJM. et al. Repair of demyelinated lesions by transplantation of purified O-2A progenitor cells. Nature 1993;362:453-5.

20. Imitola J. Genetic programs and responses of neural stem/progenitor cells during demyelination: potential insights into repair mechanisms in multiple sclerosis. Physiol. Genomics 2003;14:171-97.

21. Johansson CB, Momma S, Clarke DL, Risling M, Lendahl U, Frisen J. Identification of a neural stem cell in the adult mammalian central nervous system. Cell 2005;96:25-34.

22. John GR, Shankar SL, Shafit-Zagardo B et al. Multiple sclerosis: re-expression of a developmental pathway that restricts oligodendrocyte maturation. Nat Med 2002;8:1115-21.

23. Keirstead HS, Blakemore WF. Identification of post-mitotic oligodendrocytes incapable of remyelination within the demyelinated adult spinal cord. J Neuropathol Exp Neurol 1997:56:1191-201.

24. Kocsis JD, Akiyama Y, Radtke Ch. Neural precursors as a source to repair the demyelinated spinal cord. J Neurotrauma 2004;21:441-9.

25. Linker RA, Maurer M, Gaupp S.et al. CNTF is a major protective factor in demyelinating CNS diseases. Nat Med 2002;8:620-4

26. Lipson AC, Widenfalk J, Linquist E, Ebendal T, Olson L. Neurotrophic properties of olfactory ensheathing glia. Exp Neurol 2003;180:167-71.

27. Mason JL, Suzuki K, Chaplin DD, Matsushima GK. Interleukin -1beta promotes repair of the CNS. J Neurosci 2001;21:7046-52.

28. Mokrý J. Neurální prekurzorové buňky a jejich kultivace. Galén 1999;18

29. Nistor GI, Totoiu MO, Haque N, Carpenter MK, Keirstead HS Human embryonic stem cells differentiate into oligodendrocytes in high purity and myelinate after spinal cord transplantation. Glia 2005;49:385-96.
30. Pluchino S.et al. Injection of adult neurospheres induces recovery in a chronic model of multiple sclerosis. Nature 2003;422:688-94.

31. Pluchino S, Quattrini A, Brambilla E et al. Cell based remyelinating therapies in multiple sclerosis:evidence from experimental studies. Curr Opin Neurol 2004; 17:247-55.

32. Power J, Mayer-Proschel M, Smith J, Noble M. Oligodendrocyte precursor cells from different brain regions express divergent properties consistent with the differing time courses of myelination in these regions. Dev Biol 2002;245:362-75.

33. Prineas JW, Bernard RO, Kwon EE, Sharer LR, Cho ES. Multiple sclerosis: remyelination of nascent lesions. Ann Neurol 1993;33:137-51.

34. Ridet JL, Malhotra SK, Privat A, Gage FH. Reactive astrocytes: cellular and molecular cues of biological functions. Trends Neurosci 1997;20:570-7.

35. Ruffini F, Furlan L, Poliani PL et al. Fibroblast growth factor-II gene therapy reverts the clinical course and the pathological signs of chronic experimental encephalomyelitis in C57BL/6 mice. Gene Ther 2001;8:1207-13.

36. Smith P, Lakatos A, Barnett SC, Jeffery ND, Franklin RJ. Cryopreserved cells isolated from the adult canine olfactory bulb are capable of extensive remyelination following transplantation into the adult rat CNS. Exp Neurol 2002;176: 402-6.

37. Teng YD, Lavik EB, Qu X et al. Functional recovery following traumatic spina cord injury mediated by a unique polymer scaffold seeded with neural stem cells. Proc Natl Acad Sci USA 2002;99:3024-9.

38. Tourbah A., Linnington C, Bachelin C, Avellana-Adalid V, Werkerle H, Baron van-Evercooren A. Inflammation promotes survival and migration of the CG4 oligodendrocyte progenitors transplanted in the spinal cord of both inflammatory and demyelinated EAE rats. J Neurosci Res 1997;50:853-67.

39. Van der Goes A., Dijkstra CD. Models for demyelination. Progress in Brain Res 2001;132:149-63.

40. Viehover A, Miller RH, Park SK, Fischbach G, Vartanian T. Neuregulin: an oligodendrocytes growth factor absent in active multiple sclerosis lesions. Dev Neurosci 2001;23:377-86.

41. Webster HD. Growth factors and myelin regeneration in multiple sclerosis. Mult scler 1997;3:113-20.

42. Wewetzwr K, Verdú E, Angelov DN, Navarro X. Olfactory ensheathing glia and schwann cells: two of a kind? Cell Tissue Res 2002;309:337-45.

43. Yanai J, Doetchman T, Laufer $\mathrm{N}$ et al. Embryonic cultures but not embryos transplanted to the mouse's brain grow rapidly without immunosupression. Int J Neurosci 1995;81:21-6.

Submitted September 2005.

Accepted March 2006.

MUDr. Jan Pazour,

Neklanova 826,

46014 , Liberec,

Czech Republic.

e-mail: jan.pazour@nemlib.cz 